\title{
Process Window for Seeded Growth of Arrays of Quasi-Spherical Substrate-Supported Au Nanoparticles
}

\author{
Björn Landeke-Wilsmark, Leif Nyholm, and Carl Hägglund*
}

Cite This: Langmuir 2021, 37, 6032-6041

Read Online

ABSTRACT: The controlled growth of surface-supported metal nanoparticles (NPs) is essential to a broad range of applications. To this end, we explore the seeded growth of highly ordered arrays of substrate-supported Au NPs through a fully orthogonal design of experiment (DoE) scheme applied to a reaction system consisting of $\mathrm{HAuCl}_{4}$, citrate, and hydrogen peroxide. Scanning electron microscopy in combination with digital image analysis (DIA) is used to quantitatively characterize the resultant NP populations in terms of both particle and array features. The effective optical properties of the NP arrays are additionally analyzed using spectroscopic ellipsometry (SE), allowing characteristics of the localized surface plasmon resonances (LSPRs) of the arrays to be quantified. We study the dependence of the DIA- and SE-extracted

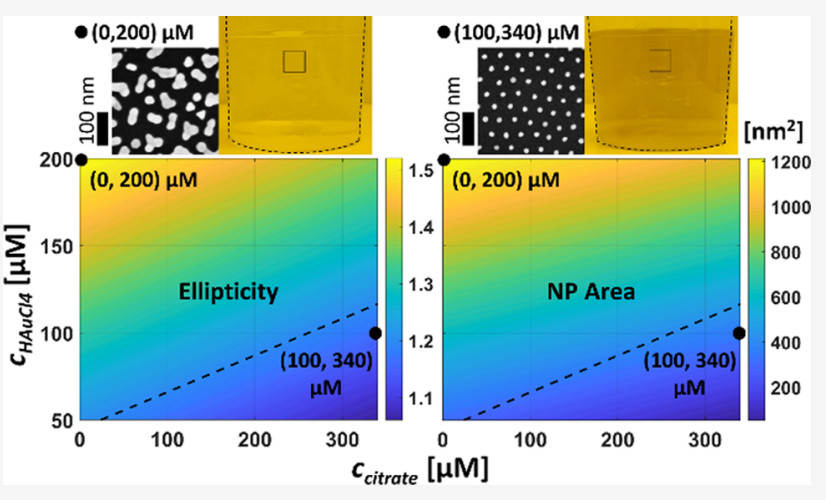
features on the different reagent concentrations through modeling using multiple linear regression with backward elimination of independent variables. A process window is identified for which uniform arrays of quasi-spherical Au NPs are grown over large surface areas. Aside from reagent concentrations the system is highly sensitive to the hydrodynamic conditions during the deposition. This issue is likely caused by an Au precursor mass-transport limitation of the reduction reaction and it is found that agitation of the growth medium is best avoided to ensure a macroscopically even deposition. Parasitic homogeneous nucleation can also be a challenge and was separately studied in a full DoE scheme with equivalent growth media but without substrates, using optical tracking of the solutions over time. Conditions yielding quasi-spherical surface-supported NPs are found to also be affiliated with strong tendencies for parasitic homogeneous nucleation and thereby loss of $\mathrm{Au}$ precursor, but addition of polyvinyl alcohol can possibly help alleviate this issue.

\section{INTRODUCTION}

Nanoparticles (NPs) composed of free-electronlike metals, such as $\mathrm{Ag}, \mathrm{Au}$, and $\mathrm{Al}$, are of great scientific and technological interest. For example, their ability to support localized surface plasmon resonances (LSPRs) with strong near-field enhancement and generation of hot carriers stimulates interest in a wide range of diverse disciplines including photocatalysis, ${ }^{1,2}$ biosensors, ${ }^{3}$ surface enhanced Raman spectroscopy, ${ }^{2,4}$ and photovoltaics. $^{5-7}$ These applications depend sensitively on the shape, size, and dielectric environment of the particles. The ability to effectively tailor these properties is therefore critical and it is often desirable to do this for NPs in surface-supported large-area arrays.

There are multiple fabrication techniques for implementing such arrays including direct-write methods like electron beam lithography ${ }^{8}$ or nanoimprint lithography, ${ }^{9}$ various colloidal self-assembly schemes, ${ }^{10,11}$ micro-/nanocontact printing, ${ }^{12}$ block copolymer (BCP) lithography, ${ }^{13-16}$ and BCP micellar lithography. ${ }^{17-19}$ Here, we utilize BCP lithography as it offers a facile and inexpensive way for rapid and parallel patterning of large surfaces with highly ordered arrays of nonclose-packed $\mathrm{Au}$ NPs of uniform size and shape, thus making it an attractive option for implementing these structures in a scalable way. However, the referenced version of BCP lithography has two drawbacks in this context: first, when using a simple (and thus low-cost) polymer chain geometry, the particle size and interparticle distance cannot readily be decoupled to any great extent. Second, due to practical limitations, the generated particles tend to be fairly small (diameter $\leq 10 \mathrm{~nm}$ ). Fortunately, both of these issues can be resolved by introducing a subsequent seeded growth step, which entails the selective deposition of additional metal on pre-existing seed particles.

Seeded growth of substrate-supported NPs comes, however, with its own set of challenges. These include (i) avoiding seed particle desorption in the case of weak particle-substrate

Received: March 12, 2021

Revised: April 13, 2021

Published: May 3, 2021 


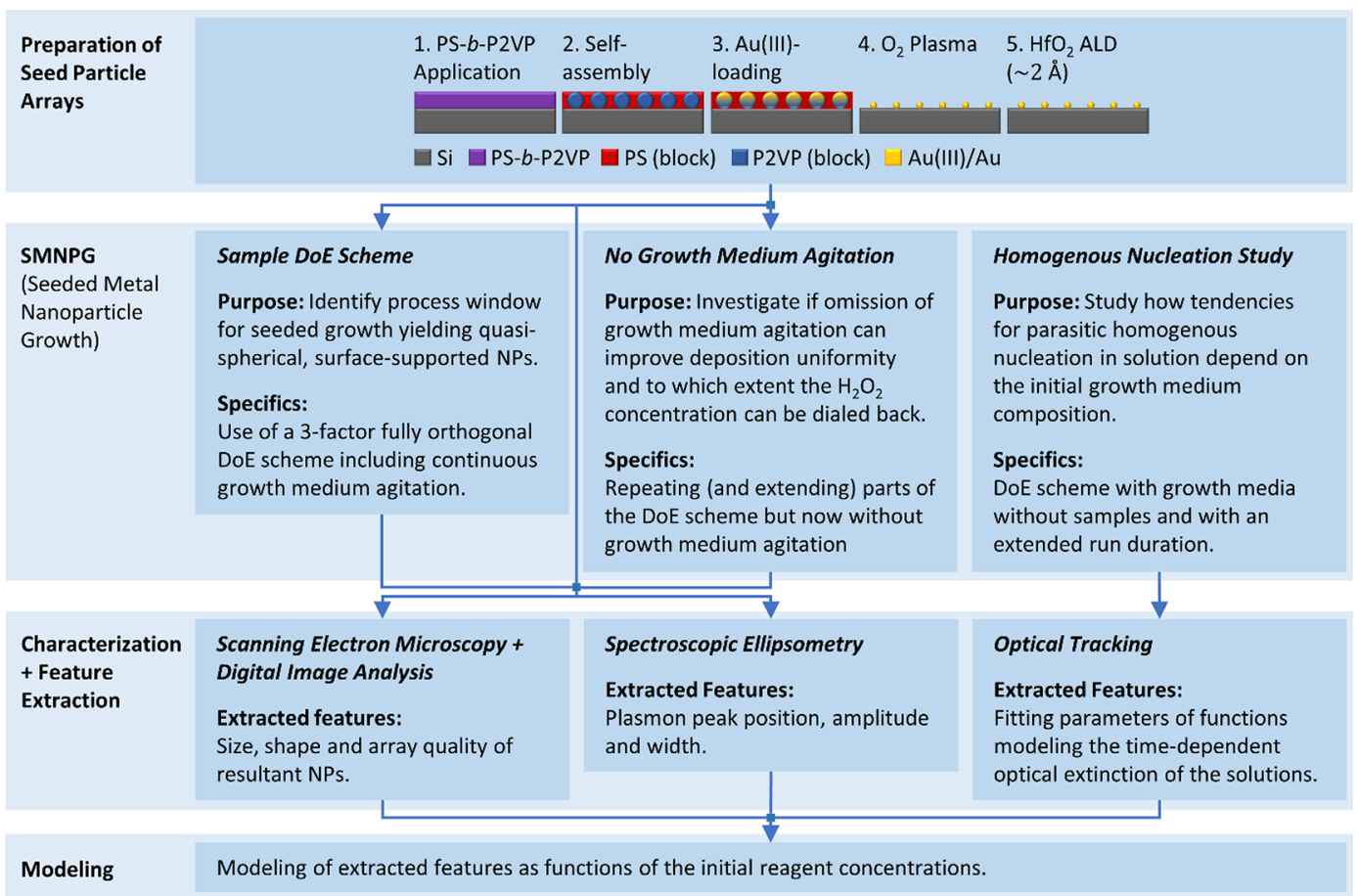

Figure 1. Overview of the experimental work and analysis included in this study. Blue arrows signify the direction of different process flows.

adhesion, (ii) achieving growth selectivity between surfaces of different materials, (iii) avoiding parasitic homogeneous nucleation (HN) in the growth medium, and (iv) if necessary, ensuring uniform hydrodynamic conditions over the sample surface during growth. As previously demonstrated, the use of atomic layer deposition (ALD) post-seed formation can be used to satisfactorily address (i). Performing as little as a single ALD cycle of $\mathrm{HfO}_{2}$ was proven to be highly effective in preventing particle desorption without hampering subsequent metal deposition. ${ }^{20}$

The concept of selective, electroless deposition of $\mathrm{Au}$ or $\mathrm{Ag}$ on BCP-derived arrays of surface-supported Au seed particles, with the aim of enlarging the $\mathrm{Au}$ seeds ${ }^{21-25}$ or implementing core-shell nanostructures, ${ }^{15,21,26,27}$ has been demonstrated previously. In the studies concerning $\mathrm{Au}, \mathrm{Au}(\mathrm{III})$ was reduced to $\mathrm{Au}(0)$ using either hydroxylammonium chloride $\mathrm{c}^{21,22}$ or a photochemical process involving UV light ${ }^{23-25}$ and less traditional reducing agents. As for the deposition of Ag, either Tollens' reagent ${ }^{26}$ or hydroquinone ${ }^{15}$ was used. The seeded growth of surface-supported arrays of Au@PNIPAM core-shell $\mathrm{NPs}^{11}$ and arrangements of multiple $\mathrm{Au}$ NPs inside larger polymer domains ${ }^{28,29}$ has also been demonstrated using a combination of ascorbic acid and $\mathrm{CTAB}$ as a weak reducing agent and a surfactant, respectively. A lot of insights and inspiration may, however, be gained by widening the frame of reference to include the extensively explored field of seeded growth of colloidal NPs suspended in solution. Two protocols $^{30,31}$ in particular caught our attention due to their (i) demonstrated ability to generate monodisperse, quasispherical NPs with tailored sizes within a large range (17-325 $\mathrm{nm}$ in diameter), (ii) rapid one-step procedure conducted at room temperature (RT), and (iii) use of relatively cheap and benign reagents. The adaptation of the growth protocol developed by $\mathrm{Liu}$ et al., ${ }^{30}$ using $\mathrm{HAuCl}_{4}$, trisodium citrate ("citrate"), and $\mathrm{H}_{2} \mathrm{O}_{2}$, has previously been investigated by us for use on arrays of surface-supported $\mathrm{Au}$ seed particles with encouraging results. ${ }^{20}$ This work is here advanced with a more rigorous, in-depth exploration of the reagent concentration parameter space coupled with quantification of various individual and collective NP features using scanning electron microscopy (SEM), digital image analysis (DIA), and spectroscopic ellipsometry (SE). This further allows us to model these features as functions of the growth medium composition and to identify a reagent concentration process window in which uniform, quasi-spherical NPs can be obtained. Moreover, we also study the effects of growth medium agitation, condition-dependent tendencies for parasitic $\mathrm{HN}$, and how the addition of poly(vinyl alcohol) (PVA) might be used to delay this and improve $\mathrm{Au}$ precursor utilization. An overview of the present study is presented in Figure 1.

\section{EXPERIMENTAL SECTION}

2.1. Materials. Poly(styrene-block-2-vinylpyridine) (PS-b-P2VP, $M_{n}=44.0-b-18.5 \mathrm{~kg} \cdot \mathrm{mol}^{-1}$, PDI $\left.=1.07\right)$ and homopolystyrene $(h \mathrm{PS}$, $M_{n}=12.5 \mathrm{~kg} \cdot \mathrm{mol}^{-1}$, PDI $=1.04$ ) were purchased from Polymer Source Inc., Canada. PS- $b$-P2VP and $h \mathrm{PS}$ were dissolved in toluene (Selectipur, Merck), while acetone (GPR Rectapur, VWR) and 2propanol (IPA, GPR Rectapur, VWR) were used for sample cleaning. Hydrogen tetrachloroaurate(III) trihydrate $\left(\mathrm{HAuCl}_{4} \cdot 3 \mathrm{H}_{2} \mathrm{O}\right.$, ACS reagent, $\geq 49.0 \% \mathrm{Au}$ basis, Fluka), trisodium citrate dihydrate $\left(\mathrm{Na}_{3} \mathrm{C}_{6} \mathrm{H}_{5} \mathrm{O}_{7} \cdot 2 \mathrm{H}_{2} \mathrm{O}, \geq 99 \%\right.$, Alfa Aesar), hydrogen peroxide $\left(\mathrm{H}_{2} \mathrm{O}_{2}, 31 \%\right.$, VLSI Selectipur, BASF $)$, PVA $\left(M_{w} \sim 31 \mathrm{~kg} \cdot \mathrm{mol}^{-1}\right.$, Mowiol 4-88, Sigma-Aldrich), deionized (DI) water, and $\mathrm{Si}(100)$ substrates $\left(\mathrm{SSP}, \mathrm{n}^{++}\right)$were used in various seeded growth procedures. Aqua regia was prepared using hydrochloric acid ( $\mathrm{HCl}, 36 \%$ VLSI Sel., BASF) and nitric acid $\left(\mathrm{HNO}_{3}, 69 \%\right.$ VLSI Selectipur, BASF).

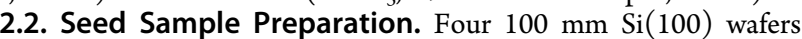
were spin-rinsed using acetone and IPA sequentially and then ashed (TePla 300, $5 \mathrm{~min}, 100 \mathrm{~W}, 50 \mathrm{sccm} \mathrm{N}_{2}+50 \mathrm{sccm} \mathrm{O} \mathrm{O}_{2}$ ) prior to subjecting them to surface functionalization with hexamethyldisilazane. This entailed $\sim 30 \mathrm{~min}$ vapor-grafting of the species at $150{ }^{\circ} \mathrm{C}$ under rough vacuum. Next, BCP films were applied via spin-coating a $0.9 \%(\mathrm{w} / \mathrm{w})$ toluene solution at $6000 \mathrm{rpm}$ to achieve a film thickness 
of approximately $21 \mathrm{~nm}$. The polymer films were composed of $30 \%$ $(\mathrm{w} / \mathrm{w}) h \mathrm{PS}_{12.5 \mathrm{k}}$ in addition to the $\mathrm{PS}_{44 \mathrm{k}}-b-\mathrm{P}_{2} \mathrm{VP}_{18.5 \mathrm{k}} \mathrm{BCP}$ to actively push the system into a sphere-forming geometry during the subsequent self-assembly step. The wafers were sequentially subjected to solvent vapor annealing (SVA) using toluene vapors in a custombuilt, flow-based setup in which the partial pressure of toluene $\left(p_{\text {tol }}\right)$ can be rapidly and dynamically controlled. The SVA was conducted at RT $\left(21{ }^{\circ} \mathrm{C}\right)$ for $1 \mathrm{~h}$ using a steady-state nominal $p_{t o l}$ of $97 \%$ of its vapor pressure $\left(p_{t o l}^{*}\right.$, i.e., $\left.p_{t o l} / p_{t o l}^{*}=0.97\right)$. The wafers were then immersed in a $2.5 \mathrm{mM} \mathrm{HAuCl}_{4}$ (aq) solution for $15 \mathrm{~min}$ to selectively load the P2VP domains with $\mathrm{AuCl}_{4}^{-}$. Next, the wafers were ashed in a two-step procedure (TePla 300, I: $5 \mathrm{~min}, 100 \mathrm{~W}, 50 \mathrm{sccm} \mathrm{O} \mathrm{O}_{2}+50$ sccm $\mathrm{N}_{2}$; II: $5 \mathrm{~min}, 1000 \mathrm{~W}, 50 \mathrm{sccm} \mathrm{O}_{2}$ ). This simultaneously removed the polymers and reduced $\mathrm{Au}(\mathrm{III})$ to $\mathrm{Au}(0)$, thereby generating highly ordered arrays of small Au NPs located at the lattice points of the previous P2VP domains in the BCP template. One ALD cycle of $\mathrm{HfO}_{2}$ (Picosun R-200, $170{ }^{\circ} \mathrm{C}, 1$ cycle: $5 \mathrm{~s} \mathrm{H}_{2} \mathrm{O}, 10 \mathrm{~s} \mathrm{~N}_{2}, 0.1 \mathrm{~s}$ tetrakis(dimethylamido)hafnium(IV), $10 \mathrm{~s} \mathrm{~N}_{2}$. Termination: $0.1 \mathrm{~s}$ $\mathrm{H}_{2} \mathrm{O}, 10 \mathrm{~s} \mathrm{~N}_{2}, 5 \mathrm{~s} \mathrm{H}_{2} \mathrm{O}$ ) was performed on all wafers, to augment the particle-substrate adhesion, ${ }^{20}$ prior to applying (spin-coating, 500 rpm) and soft-baking (hotplate, $110{ }^{\circ} \mathrm{C}, 10 \mathrm{~min}$ ) a thick layer of S1813 photoresist. The purpose of the $\mathrm{S} 1813$ is to serve as a protective coating during the subsequent dicing (Disco DAD 361) of the wafers into $20 \times 20 \mathrm{~mm}^{2}$ sample pieces. Several smaller edge pieces, in addition to the nine appropriately sized samples, were obtained per wafer. The S1813 was then stripped using acetone, IPA, and additional ashing (TePla 300, $5 \mathrm{~min}, 50 \mathrm{~W}, 50 \mathrm{sccm} \mathrm{O}_{2}+50$ sccm $\mathrm{N}_{2}$ ). Although one ALD cycle of $\mathrm{HfO}_{2}$ can boost the particle adhesion sufficiently, a second ALD cycle was lastly performed to immobilize any dislodged NPs in the immediate vicinity of the dicing cuts. Structure verification using SEM was performed after the precursor-loading of the BCP template and after the last ALD step.

2.3. Design of Experiment (DoE) Scheme for Seeded Metal Nanoparticle Growth. The procedure for seeded metal nanoparticle growth (SMNPG) was conducted by immersing individual samples, affixed to disposable sample mounts of polystyrene (PS), in freshly prepared growth media in disposable polypropylene (PP) beakers for $5 \mathrm{~min}$. The samples were attached to the PS mounts using a hotglue resin, which is believed to be inert under these conditions. The PP beakers and PS sample mounts were copiously rinsed with IPA and DI water prior to use. Upon process termination, the samples were removed from the solution, thoroughly rinsed with DI water and IPA, and dried with a $\mathrm{N}_{2}$ gun. While immersed, the samples were oriented vertically at the radial edge of the beaker and with the NP-decorated surface facing the center of the vessel. As PTFE-clad magnetic stirring bars were used to generate a mild vortex in the growth medium, a directed flow over the decorated surface was achieved. The risk of cross-contamination between procedures was minimized through the use of a new set of disposable labware and aqua regia-cleaned [3:1 $\left.\mathrm{HCl}(36 \%): \mathrm{HNO}_{3}(69 \%)\right]$ stirring bar for each sample. (Caution! Aqua regia solutions are strongly corrosive and oxidizing; adding organics may cause an explosion).

To investigate the effects of the reagent concentrations (i.e., $c_{\mathrm{HAuCl} 4}$, $c_{\text {citrate }}$, and $c_{\mathrm{H}_{2} \mathrm{O} 2}$ ), a fully orthogonal 3 -factor DoE scheme, including three centerpoint (CP) replicates, was implemented with levels as shown in Figure 2. The total volume $(40 \mathrm{~mL})$ was kept constant and the reagents were added in the following order: DI water, citrate, $\mathrm{HAuCl}_{4}, \mathrm{H}_{2} \mathrm{O}_{2}$, and lastly the seed-decorated sample. The stock solutions used were $50 \mathrm{mM} \mathrm{HAuCl}_{4}(\mathrm{aq}), 1 \%(\mathrm{w} / \mathrm{w})$ citrate $(\mathrm{aq})$, and $31 \%(\mathrm{w} / \mathrm{w}) \mathrm{H}_{2} \mathrm{O}_{2}(\mathrm{aq})$. The DoE runs were all conducted at RT (21 ${ }^{\circ} \mathrm{C}$ ) and under yellow light conditions over a span of three consecutive days in a fume hood in a climate-controlled cleanroom environment. To minimize unintentional biasing, the run order and the parent wafer sample origin (i.e., from which one of the four parent wafers of the sample was taken) were randomized. The only constraint imposed regarding the sample origin was that only nine samples could be drawn from any one parent wafer.

2.4. DoE Scheme for Growth Media without Samples. To investigate the tendency for $\mathrm{HN}$ as a function of growth conditions, the entire DoE scheme was repeated using an extended run duration

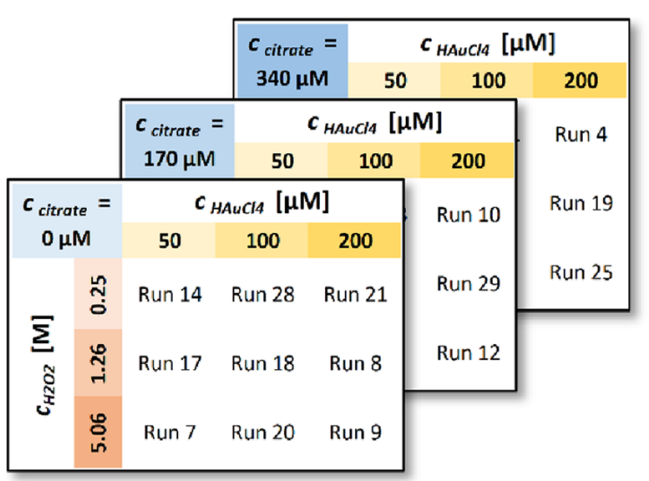

Figure 2. DoE scheme (CP replicates not shown). For clarity, the combinations of $c_{\text {citrate }}$ and $c_{\mathrm{H} 2 \mathrm{O} 2}$ are the same for all three levels of $c_{\text {citrate }}$

of $15 \mathrm{~min}$ and growth media without immersed samples or sample holders. These runs were conducted over a span of two consecutive days with equipment and experimental conditions otherwise identical to those in the original SMNPG DoE scheme.

2.5. Growth without Agitation. In addition to the two DoE schemes, a follow-up experiment was also conducted with the aim to elucidate whether the macroscale deposition uniformity could be improved by omitting the growth medium agitation. The experiment consisted of a series of SMNPG procedures using a progressively decreasing $c_{\mathrm{H} 2 \mathrm{O} 2}$. The experimental setup was identical to that used in the sample DoE scheme except that instead of using a stirring bar, the reagents were rapidly mixed (upon $\mathrm{H}_{2} \mathrm{O}_{2}$ addition) by drawing the solution once in and out of a $10 \mathrm{~mL}$ micropipette prior to sample immersion. The immersion time was also extended from 5 to $10 \mathrm{~min}$ to mitigate the effects of a less efficient reagent mass transport.

2.6. Characterization of DoE Samples. SEM images were acquired at three specific sample inspection points (IP1-3) using a Zeiss 1530 SEM $\left(V_{a c c}=5 \mathrm{kV}, 0^{\circ}\right.$ stage tilt, WD 3 mm, in-lens SEII detector) post-SMNPG. IP1-3 lie along one of the sample diagonals with IP1 and IP3 closer to the corners, while IP2 is in the sample center (Figure S2b). For reference, the seed particle arrays on two random samples from each parent wafer were inspected prior to the SMNPG.

DIA of one SEM image, acquired at $200 \mathrm{k}$ magnification, was performed for each inspection point of the samples using CellProfiler 3.0 software. In brief, a small sweeping median filter $(3 \times 3$ pixel $)$ was used for initial smoothing and noise reduction prior to object identification using the minimum cross-entropy thresholding algorithm. ${ }^{32}$ Purposefully, no attempt at declumping fused or touching particles was made and NPs located on the image border were excluded from the number count $(N)$ and size and shape characterization but included when estimating $\phi$ (defined below). The DIA-extracted parameters include the relative change in NP count $\left(\Delta N / N_{0}\right)$, where $N_{0}$ is obtained from the corresponding wafer reference samples, surface area fraction covered by NPs $(\phi)$, mean values of the NP top-view cross-sectional area $\left(\bar{x}_{\text {Area }}\right)$, maximum Ferret diameter $\left(\bar{x}_{\text {Max FD }}\right)$, form factor $\left(\bar{x}_{F F}\right)$, and ellipticity $\left(\bar{x}_{\text {Ellips }}\right)$ as well as their corresponding SDs $\left(s_{x}\right)$. The form factor (FF) is defined as $4 \pi \cdot$ area/perimeter ${ }^{2}$ and equals 1 for NPs with perfectly circular top-view cross sections. The ellipticity on the other hand is the ratio of the major to minor axes of an ellipse fitted to the NP cross section. $s_{d}$ is the SD of the centroid-to-centroid distance between the NPs and their nearest neighbor. $s_{\theta}$ is the SD of the discrepancy between the angle spanned by the centroids of the two nearest neighbors, with the apex in the centroid of the NP under study, and that of an ideal hexagonal array pattern; essentially, $n \cdot 60^{\circ}$ is subtracted from the measured angle where $n$ is the rounded-off integer of the measured angle divided by $60^{\circ} \cdot \bar{x}_{\text {Area }}$ and $\bar{x}_{\text {Max FD }}$ are NP size indicators, $\bar{x}_{F F}$ and $\bar{x}_{\text {Ellips }}$ are shape features, whereas $s_{d}$ and $s_{\theta}$ to some extent indicate how well the array pattern is maintained after the SMNPG. $\Delta N / N_{0}, S_{d}$ and $S_{\theta}$ are, however, dependent on both the size and shape of the NPs 
as well as the quality of the array pattern. $\Delta N / N_{0}$ will be further affected by the orientation of the SEM acquisition area in relation to the array lattice.

SE measurements [Woollam RC2-XI, photon energy $(E)$ range $=$ $[0.7,4.4] \mathrm{eV}$, angle of incidence $\left.65^{\circ}\right]$ were conducted in the center of the DoE samples with the probe spot along the diagonal running through points IP1-3. Au NP arrays on a substrate surface can optically be described in terms of an effective medium with an effective layer thickness $\left(t_{\text {eff }}\right)$ and relative permittivity $\left(\epsilon=\epsilon_{1}+i \epsilon_{2}\right)$. $t_{e f f}$ and $\epsilon$ were estimated by fitting an oscillator model, consisting of a PSemi-M0, a PSemi-Tri, and $\leq 3$ Gaussian oscillators, to the SE measurement data. In $\epsilon_{2}$, the background is represented by the PSemi$\mathrm{MO}$ and a wide Gaussian centered at high $(>4 \mathrm{eV})$ photon energies (E) while the PSemi-Tri and remaining Gaussians correspond to the LSPR. The LSPRs of the NP arrays were characterized using automated peak detection [the findpeaks function] in MATLAB on the $\epsilon_{2}$ contribution of the sum of the LSPR oscillators. The extracted features were the LSPR peak position $\left(E_{L S P R}\right)$, amplitude $\left(\epsilon_{2, L S P R}\right)$, and full width at half maximum (FWHM). Two seed particle array reference samples per parent wafer were equally analyzed for comparison.

2.7. Characterization of the Growth Media without SeedParticle Arrays. During the unseeded DoE scheme, the growth media were recorded using a digital camera setup. Image frames were converted to grayscale and analyzed at a $2 \mathrm{~s}$ interval, starting $20 \mathrm{~s}$ after $\mathrm{H}_{2} \mathrm{O}_{2}$ addition to allow reagent mixing to complete. The mean pixel intensity $(I)$ was then calculated in a fixed image window centered on the transparent PP beaker. To track changes over time and enable cross-sample comparisons, the relative intensity change $\Delta I(t) / I_{0} \equiv$ $[(I(t=0 \mathrm{~s})-I(t)) / I(t=0 \mathrm{~s})]$ was calculated. Functions of the form $f(t)=A+K\left\{1+\exp \left[-\left(t-t_{0}\right) / \tau\right]\right\}^{-1}$ were then fitted to the $\Delta I(t) /$ $I_{0}$ data, with $A, K, \tau$, and $t_{0}$ as simultaneous fitting parameters.

2.8. Characterization of Samples Grown without Agitation. These samples were characterized equivalently to those in the sample DoE scheme with two exceptions. First, the DIA pipeline was modified so as to analyze and pool the results of two images acquired at $400 \mathrm{k}$ magnification per inspection site. Second, multiple angles of incidence were used (i.e., $\{65,70 \text {, and } 75\}^{\circ}$ ) during the SE data acquisition.

2.9. Statistical Modeling of Extracted Features. Multiple linear regression (MLR) with backward elimination ${ }^{33}$ (significance level, $\alpha=0.05$ to exclude) of independent variables was performed for several of the extracted features using the statistical software Minitab 17. This method entails an automated, iterative modeling procedure starting with the full model containing the reagent concentrations $c_{\text {citrate }}, c_{\mathrm{HAuCl} 4}$, and $c_{\mathrm{H} 2 \mathrm{O} 2}$ as well as their interaction and second order terms. Sequentially, the least significant variable, that is, the one with the highest $p$-value, is then eliminated one at a time until all remaining variables have a $p$-value smaller than $\alpha .^{33}$ No boundary conditions were imposed, and the independent variables were standardized (i.e., subtraction of the mean and division with the SD were performed) prior to modeling to reduce effects of multicollinearity. ${ }^{33}$ In instances of $R^{2}<80 \%$ ( $R^{2}$ defined below) and non-normally distributed residuals, an MLR was also conducted on the natural logarithm of that response variable. If substantial improvement was obtained, the model with the transformed response was adopted. When modeling the DIAextracted features, the seed particle reference samples were only included in the dataset for $s_{d}$ and $s_{\theta}$ (see definitions above) as the SEM images of the references were acquired at a lower $V_{\text {acc }}(1 \mathrm{kV}) . R^{2}$ (ratio of explained variance to total variance), adjusted $R^{2}\left(R^{2}\right.$ adjusted for the degrees of freedom in the model), and $R_{\text {pred }}^{2}$ (a measure of the predictive strength of the model) are the goodness of fit parameters used to quantify the fit and applicability of the models. ${ }^{33}$ The $R^{2}$ contribution of each independent variable included in the model to the total $R^{2}$ is also given to highlight its importance to the model.

\section{RESULTS AND DISCUSSION}

3.1. DoE Scheme for SMNPG. The SEM structure verification of the loaded BCP templates reveals highly ordered patterns of hexagonally, nonclose-packed, $\mathrm{AuCl}_{4}{ }^{-}$-loaded $\mathrm{P} 2 \mathrm{VP}$ domains in a PS matrix (Figure S1a-h). Subsequent ashing of these structures generates highly ordered Au seed particle arrays on the Si substrate (Figure S1i-q) with NPs of uniform size and shape (Table S1). The average values of the maximum Ferret diameter $\left(\bar{x}_{\text {maxFD }}\right)$, ellipticity $\left(\bar{x}_{\text {Ellips }}\right)$, and center-tocenter interparticle distance $\left(\bar{x}_{d}\right)$ are approximately $9,1.1$, and $41 \mathrm{~nm}$, respectively. $\bar{x}_{\text {Ellips }}$ is likely an overestimate though, due to the small NP size and finite pixel resolution. A small fraction of vacant lattice positions can be seen on the reference samples originating from one of the parent wafers (Figure S1j,n), possibly due to defects in the BCP template or insufficient precursor access during the loading procedure. The areal seed particle density on this wafer is $5.5 \%$ lower than the average of fully covered wafers, but out of the image-extracted features, this is only expected to affect the fractional areal coverage $(\phi)$ in any significant way.

Seeded growth with agitation results in a macroscopically uneven deposition, as evident from visual inspection (Figure S2a), comparison of the SEM images (Figures S3-S5), and the affiliated DIA-extracted feature values (Table S2, Section I and Table S3). For example, taking only the DoE CP replicates into account, the average cross-sectional area $\left(\bar{x}_{\text {area }}\right)$ of the NPs in the opposite corner points (IP1 and IP3) is $73 \%$ higher and $32 \%$ lower, respectively, than that in the middle point (IP2). $\mathrm{An} \mathrm{Au}$ precursor mass-transport limitation of the deposition process in combination with growth medium agitation is the likely cause considering the vastly lower concentration of $\mathrm{HAuCl}_{4}$ compared to $\mathrm{H}_{2} \mathrm{O}_{2}$ throughout the DoE scheme. Such a mass-transport limitation would make the deposition highly sensitive to the local hydrodynamic conditions, which is consistent with the deposition pattern observed here. Drawing meaningful conclusions regarding the dependencies of the DIA- and SE-extracted features on the reagent concentrations is, nonetheless, still believed to be possible by exclusively analyzing the results obtained in the middle inspection point (IP2). The hydrodynamic conditions ought to vary the least between the samples here. The SE measurement spot was centered on this point. A compilation of SEM images from all the DoE samples shows that our DoE scheme covers the generation of NPs of various sizes, shapes, and degrees of interconnectedness. This suggests that the DoE reagent concentration ranges used were sufficiently broad here (Figure 3).

A straightforward way of probing the strength of linear relationships between the extracted features internally and the reagent concentrations is to compile a correlation matrix stating the pairwise Pearson correlation coefficients $(\rho)$ (Figure S6). A strong correlation $(|\rho| \geq 0.73)$ between $c_{\mathrm{HAuCl}}$ and all DIA-extracted features, which in turn are all strongly correlated with each other $(|\rho| \geq 0.78)$, can be observed. The positive correlations between $\phi, \bar{x}_{\text {Area }}, \bar{x}_{\text {Max FD }}$, and $c_{\mathrm{HAuCl}}$ are intuitive as the three features are all indicators of the amount of material deposited and $c_{\mathrm{HAUCl}}$ dictates the availability of the $\mathrm{Au}$ precursor. The correlations between the DIA features and $c_{\mathrm{H} 2 \mathrm{O} 2}$ are very weak $(|\rho| \leq 0.15)$, whereas those toward $c_{\text {citrate }}$ are stronger $(0.17 \leq|\rho| \leq 0.51)$ in the probed parameter space. The former is likely related to the fact that $c_{\mathrm{H} 2 \mathrm{O} 2} \gg c_{\mathrm{HAuCl} 4}$ even for the lowest $c_{\mathrm{H} 2 \mathrm{O} 2}$ DoE level used. The strong relationship 


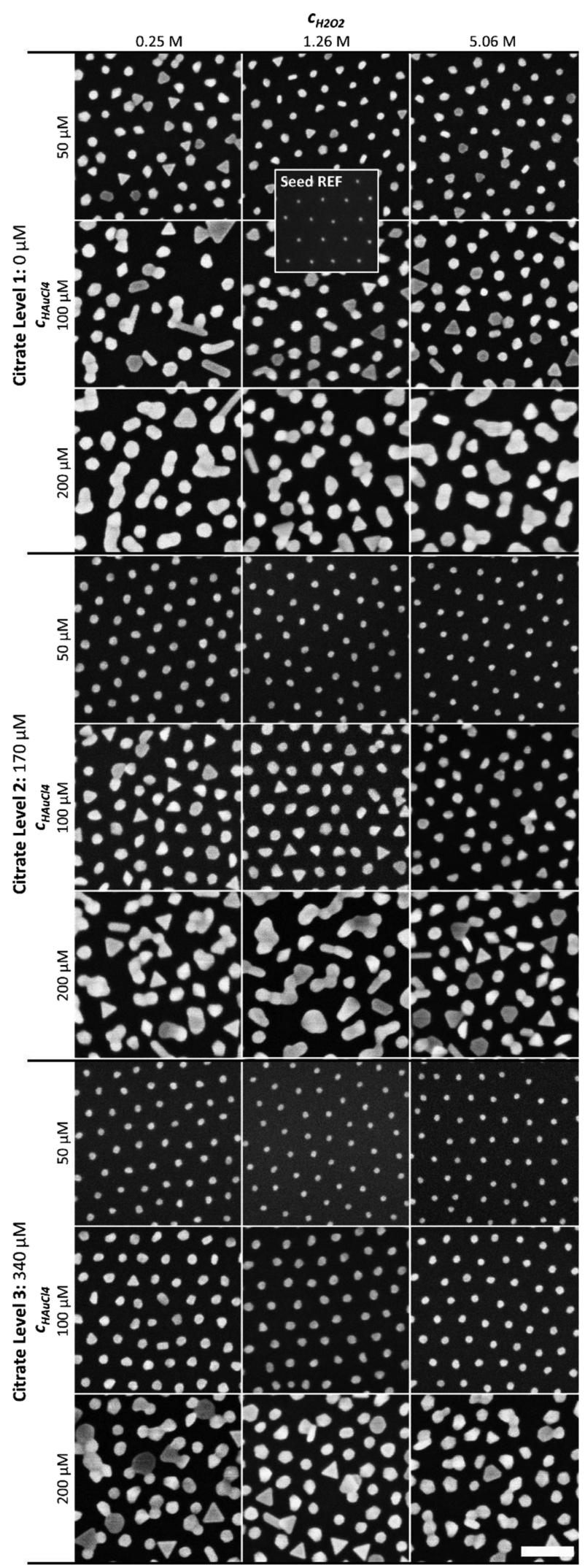

Figure 3. Compilation of SEM images acquired at the center (IP2) of the DoE samples after the SMNPG procedures. The scale bar equals $100 \mathrm{~nm}$. between the SDs $s_{\text {Area }}, s_{\text {Max FD }}, s_{F F}$, and $s_{\text {Ellips }}$ and their corresponding means implies a diversifying NP population, in terms of size and shape, with increasing growth.

To investigate the potential presence of more complex dependencies of the extracted sample features, we also performed modeling using MLR with backward elimination of independent variables on each DIA feature, as described in the Experimental Section. ${ }^{33}$ The significant independent variables, their effects, and relative importance are summarized in Table 1; the numerical values of the fitted coefficients are listed in Table S4. Decent statistical models, with adjusted explained variance ratio adj. $R^{2} \geq 76.4 \%$ and predictive strength $R_{\text {pred }}^{2} \geq 74.3 \%$, are obtained, and we observe that the $R^{2}$ contribution of $c_{\mathrm{HAuCl} 4}$ (and its square term) completely dominates in these models. $c_{\text {citrate }}$ is a significant but limitedinfluence variable in all but the model concerning the effective optical thickness. Meanwhile, $c_{\mathrm{H} 2 \mathrm{O} 2}$ only provides a marginal explanatory contribution in two of the models and is otherwise insignificant.

As many applications of Au NPs involve optics, photonics, or plasmonics, it is of great interest to also characterize the optical response of the resultant NP arrays using SE. The energy-resolved relative permittivity $\left(\epsilon=\epsilon_{1}+i \epsilon_{2}\right)$ of the effective media formed by the NP arrays (Figures S7-S11) is of particular interest as it can be used to characterize the collective LSPRs - as detailed in the Experimental Section (Table S2, Section II). The SE results are, however, likely affected by the deposition gradient across the SE measuring spot, which depending on incidence angle can extend for up to $10 \mathrm{~mm}$. Nonetheless, both correlations and MLR models were calculated for the SE-extracted effective thickness $\left(t_{\text {eff }}\right)$, the plasmon peak position $\left(E_{L S P R}\right)$, amplitude $\left(\epsilon_{2, L S P R}\right)$, and width (FWHM), with the exception of FWHM, which could not be obtained for all DoE samples due to limitations in the spectral range. The correlations between $\left\{E_{L S P R}, \epsilon_{2, L S P R}\right.$, and $\left.t_{e f f}\right\}$ and $c_{\mathrm{HAuCl} 4}$ are moderate to strong $(|\rho| \geq 0.67)$, while the corresponding values for $c_{\text {citrate }}$ and $c_{\mathrm{H} 2 \mathrm{O} 2}$ are weak $(|\rho| \leq$ $0.34)$ and very weak $(|\rho| \leq 0.11)$, respectively (Table S6). At most, marginal effects of $c_{\text {citrate }}$ and $c_{\mathrm{H} 2 \mathrm{O} 2}$ on the SE features are also suggested by the MLR models (Table 1). $c_{\mathrm{HAuCl} 4}$ on the other hand confers a strong explanatory power, which is readily understood from the fact that a higher $c_{\mathrm{HAuCl}}$ enables larger particles and thereby prominently affects all of the SE features. According to our MLR models, a higher $c_{\mathrm{HAuCl} 4}$ is affiliated with an increase of $\epsilon_{2, L S P R}$ and a decrease of $E_{L S P R}$ (i.e., a redshift of the LSPR peak), which is consistent with what would be expected, as larger NPs have a higher polarizability and support longer wavelength resonances. ${ }^{34}$ The nature of the LSPR peak can, however, be affected by more than just the size and shape distributions of the individual NPs. Some degree of modulation is also conceivable due to interparticle near-field coupling, the strength of which decays on a characteristic length scale of approximately 0.4 times the radius of the NPs. ${ }^{29}$ This phenomenon might be applicable to samples for which the initial array order is not maintained and where large, irregularly shaped NPs can be observed in close proximity, leading especially to an increased peak width in the measurements. The decay of the array order is, at least in part, likely attributable to the vigorous agitation of the growth media employed in the DoE scheme. An example of the SEanalyzed optical properties of two distinctively different NP arrays after seeded growth is shown in Figure 4. 
Table 1. Extracted Features Modeled Using Multiple Linear Regression ${ }^{a}$

\begin{tabular}{|c|c|c|c|c|c|c|c|c|c|c|c|}
\hline & \multirow[b]{2}{*}{$f(x)$} & \multicolumn{3}{|c|}{ Goodness of Fit } & \multicolumn{7}{|c|}{$R^{2}$ Contribution $^{\mathrm{a}}$} \\
\hline & & $R^{2}$ & Adj. $R^{2}$ & $R^{2}$ pred & $C_{\text {citrate }}$ & $C_{\text {HAUCl4 }}$ & $c_{\mathrm{H} 2 \mathrm{O} 2}$ & $\begin{array}{l}c_{\text {citrate }} \cdot \\
c_{\text {HAuCl4 }}\end{array}$ & $\begin{array}{c}C_{\text {citrate }} \\
C_{\mathrm{H} 2 \mathrm{O} 2}\end{array}$ & $\begin{array}{c}c_{\mathrm{HAUCl} 4^{\circ}} \\
c_{\mathrm{H} 2 \mathrm{O} 2}\end{array}$ & $c_{\mathrm{HAUCl4}}^{2}$ \\
\hline \multirow{12}{*}{ 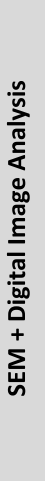 } & $\Delta \mathbf{N} / \mathbf{N}_{0}$ & $81.1 \%$ & $79.7 \%$ & $76.4 \%$ & (+) $9.5 \%$ & $(-) 71.6 \%$ & - & - & - & - & - \\
\hline & $\varphi$ & $95.0 \%$ & $94.2 \%$ & $92.9 \%$ & (-) $3.9 \%$ & (+) $85.7 \%$ & $(-) 2.5 \%$ & $(+) 2.8 \%$ & - & - & - \\
\hline & $\bar{x}_{\text {Area }}$ & $92.1 \%$ & $91.5 \%$ & $90.0 \%$ & (-) $6.7 \%$ & $(+) 85.3 \%$ & - & - & - & - & - \\
\hline & $s_{\text {Area }}$ & $91.7 \%$ & $90.7 \%$ & $88.8 \%$ & $(-) 2.8 \%$ & (-) $84.8 \%$ & - & - & - & - & (+) $4.1 \%$ \\
\hline & $\bar{X}_{\text {Max FD }}$ & $95.2 \%$ & $94.6 \%$ & $93.1 \%$ & $(-) 8.8 \%$ & $(+) 85.2 \%$ & $(-) 1.2 \%$ & - & - & - & - \\
\hline & $S_{\text {Max FD }}$ & $91.9 \%$ & $90.9 \%$ & $89.1 \%$ & $(-) 4.7 \%$ & (+) $85.1 \%$ & - & - & - & - & (+) $2.0 \%$ \\
\hline & $\overline{\boldsymbol{X}}_{\mathrm{FF}}$ & $89.9 \%$ & $89.1 \%$ & $87.1 \%$ & (+) $8.5 \%$ & $(-) 81.3 \%$ & - & - & - & - & - \\
\hline & $s_{F F}$ & $77.2 \%$ & $76.4 \%$ & $74.3 \%$ & - & (+) $77.2 \%$ & - & - & - & - & - \\
\hline & $\bar{x}_{\text {Ellip }}$ & $89.1 \%$ & $88.2 \%$ & $86.5 \%$ & (-) $15.9 \%$ & $(+) 73.1 \%$ & - & - & - & - & - \\
\hline & $S_{\text {Ellip }}$ & $86.9 \%$ & $85.9 \%$ & $83.9 \%$ & $(-) 21.7 \%$ & $(+) 65.2 \%$ & - & - & - & - & - \\
\hline & $s_{d}$ & $83.8 \%$ & $81.8 \%$ & $76.6 \%$ & $(-) 0.2 \%$ & (+) $79.6 \%$ & - & $(-) 2.6 \%$ & - & - & $(+) 1.3 \%$ \\
\hline & $s_{\theta}$ & $84.2 \%$ & $82.8 \%$ & $80.2 \%$ & $(-) 0.3 \%$ & (+) $79.7 \%$ & - & - & - & - & (-) $4.2 \%$ \\
\hline \multirow{3}{*}{ 岕 } & $\boldsymbol{t}_{\text {eff }}$ & $90.8 \%$ & $90.0 \%$ & $88.3 \%$ & (-) $1.8 \%$ & $(+) 86.6 \%$ & - & - & - & - & (-) $2.4 \%$ \\
\hline & $E_{L S P R}$ & $68.5 \%$ & $66.6 \%$ & $62.5 \%$ & $(+) 0.8 \%$ & $(-) 67.7 \%$ & - & - & - & - & - \\
\hline & $\varepsilon_{2, L S P R}$ & $89.2 \%$ & $87.9 \%$ & $84.1 \%$ & (-) $3.6 \%$ & (+) $83.7 \%$ & $(+) 0.0 \%$ & - & - & (-) $1.9 \%$ & - \\
\hline \multirow{3}{*}{ 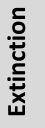 } & $\ln \left(t_{0}\right)$ & $89.1 \%$ & $87.4 \%$ & $84.7 \%$ & (-) $28.3 \%$ & (+) $30.8 \%$ & (-) $18.5 \%$ & (+) $11.6 \%$ & - & - & - \\
\hline & $\ln (\tau)$ & $87.0 \%$ & $83.6 \%$ & $73.8 \%$ & $(-) 31.9 \%$ & $(+) 29.5 \%$ & (-) $14.2 \%$ & $(+) 5.5 \%$ & $(-) 3.3 \%$ & $(+) 2.8 \%$ & - \\
\hline & $\kappa$ & $83.1 \%$ & $80.4 \%$ & $74.2 \%$ & (+) $41.1 \%$ & (-) $22.5 \%$ & - & (-) $14.8 \%$ & - & - & (+) $4.7 \%$ \\
\hline
\end{tabular}

${ }^{a} R^{2}$-contribution of the significant linear $\left(c_{\text {citrate, }}, c_{\mathrm{HAuCl} 14}\right.$ and $\left.c_{\mathrm{H} 2 \mathrm{O} 2}\right)$, interaction $\left(c_{\text {citrate }} \cdot c_{\mathrm{HAuCl} 4}, c_{\text {citrate }} \cdot c_{\mathrm{H} 2 \mathrm{O} 2}\right.$ and $\left.c_{\mathrm{HAuCl} 14} \cdot c_{\mathrm{H} 2 \mathrm{O} 2}\right)$ and square $\left(c_{\mathrm{HAuCl}}{ }^{2}\right)$ terms to the total $R^{2}$-value of the models. The contributions from all significant terms add up to the total $R^{2}$-value of the model. The sign of the coefficients are stated within the parenthesis. The reference samples are included in the modeling of $s_{\mathrm{d}}, s_{\theta}$ and the SE features.

3.2. DoE Scheme without Samples. Although the surface-supported NPs are of primary interest in this study, the tendency for $\mathrm{HN}$ in solution is important as it entails parasitic consumption of the available Au precursor and leads to reduced and less resource-efficient seeded growth. Hence, to investigate trends in the onset and severity of $\mathrm{HN}$, the full $\mathrm{DoE}$ scheme was repeated using growth media without samples and an extended run duration of $15 \mathrm{~min}$. A digital camera setup was used to optically monitor the solutions. In addition to compiling image collages of the media side-by-side at a set of specific time coordinates (Figures S12-S14), the relative change in mean grayscale pixel intensity $\left[\Delta I(t) / I_{0}\right]$ was also calculated and tracked over time $(t)$ for each run (Figure S15). $\Delta I(t) / I_{0}$ is here devised as a proxy for the combined extent of undesirable HN and growth of NPs in solution. This is motivated by the fact that suspended colloidal NPs will absorb and scatter light, causing the recorded pixel intensity to drop and $\Delta I(t) / I_{0}$ to increase commensurately. Next, $\Delta I(t) / I_{0}$ was modeled by fitting functions of the form $f(t)=A+K\{1+\exp [$ $\left.\left.-\left(t-t_{0}\right) / \tau\right]\right\}^{-1}$ to the data from each run (Figures S16-S19 and Table S2, Section III). The sigmoid functional form captures the behavior of $\Delta I(t) / I_{0}$ very well except for in a few instances of discontinuity artifacts and/or a declining asymptotic plateau. Gas evolution in the medium, stemming from the NP-catalyzed decomposition of $\mathrm{H}_{2} \mathrm{O}_{2}$, is the likely cause of these artifacts as generated bubbles of varying numbers, sizes, and residence times can cling to the beaker wall (Figures S13 and S14). Although no gas bubble formation on the seed-decorated sample surface was observed in any of our sample DoE runs, if it would occur, it might interfere with precursor access. Thus, caution in using excessively high $c_{\mathrm{H} 2 \mathrm{O} 2}$ might be warranted. The fitting parameters $t_{0}$ and $\tau$ are related to the onset and duration of the $\Delta I(t) / I_{0}$ change, respectively, whereas $A+K$ is the value of the asymptotic plateau of $f(t)$, that is, as $t \rightarrow \infty$. However, as $A$ merely accounts for very minor curve offsets, $K$ is the parameter of interest to gauge the magnitude of change. All other things being equal, it is desirable for $t_{0}$ and $\tau$ to be large while $K$ ideally should be small as this would correspond to a late onset of a slow and low magnitude change. Considering the correlation matrix (Table S6) and performing individual MLRs on $K, t_{0}$, and $\tau$ (Table 1 ) suggest that either increasing $c_{\text {citrate }}$ or decreasing $\mathcal{c}_{\mathrm{HAuCl} 4}$ will cause a larger $\Delta I(t) / I_{0}$ change, which further will start earlier and occur faster. A high $c_{\text {citrate }}: c_{H A u C l 4}$ ratio, moreover, results in blueish growth media, whereas more reddish solutions are obtained for lower ratios (Figures S12-S14). This is interpreted as a high $c_{\text {citrate }}: c_{\mathrm{HAuCl} 4}$ ratio being affiliated with more rapid and extensive $\mathrm{HN}$, resulting in a higher number of small but growing colloidal NPs contributing to the optical extinction of the medium. This is the opposite of what we would expect merely on the basis of thermodynamics as the tendency for $\mathrm{HN}$ should increase with increasing redox potential $\left(E_{r}\right)$ of the system. The dependencies of $E_{r}$ on the reagent concentrations are given by the Nernst equation, $E_{r}=E_{r}^{o}-\frac{\mathrm{RT}}{\mathrm{zF}} \ln Q_{r}$, where $E_{r}^{o}, R, T, z, F$, and $Q_{r}$ are the standard redox potential, universal gas constant, temperature, number of electrons transferred in the reaction, Faraday constant, and reaction quotient, respectively. As $c_{\mathrm{HAuCl} 4}$ occurs in the denominator of $Q$, an increase ought to lead to a higher $E_{r}$. The addition of citrate, on the other hand, is expected to mainly affect $\mathrm{Au}(\mathrm{III})$ complex speciation by acting as an alternative ligand to $\mathrm{Cl}^{-}$, thereby lowering $E_{r}$. As both trends are at odds with our observations, we thus conclude that the 
a)
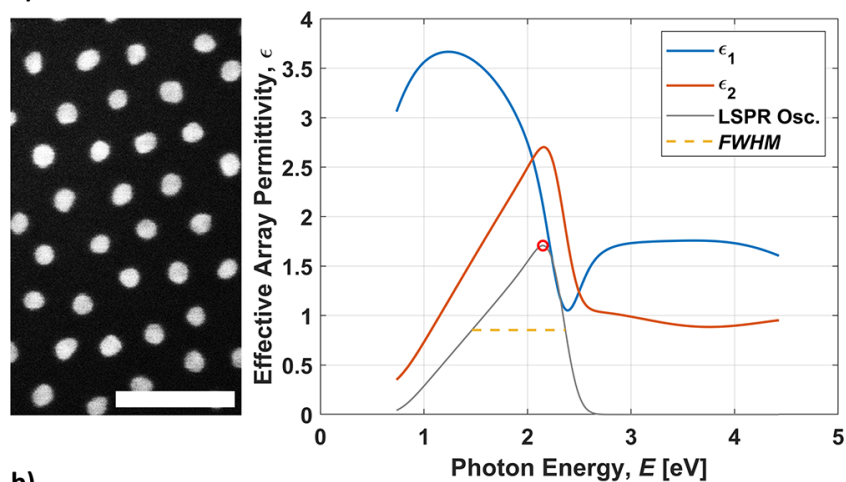

b)

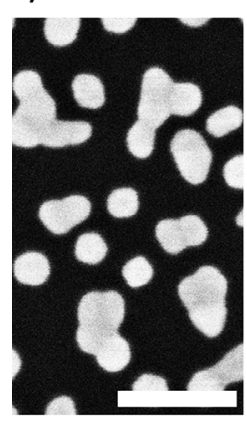

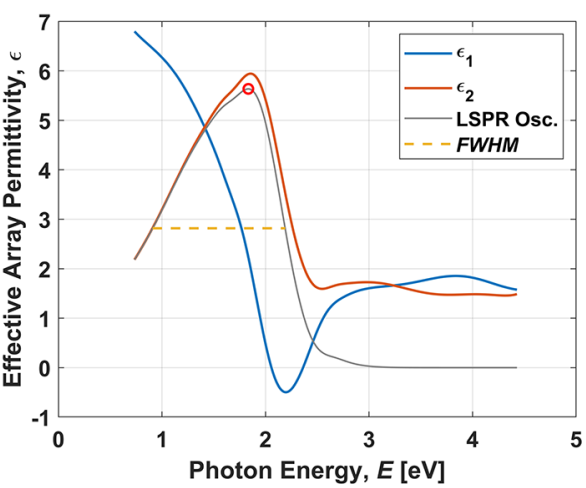

Photon Energy, $E[\mathrm{eV}]$

Figure 4. SEM images and plots of the effective array permittivity $\epsilon$ from two representative samples post-SMNPG with (a) moderately sized well-separated NPs and (b) large polydisperse NPs. The effective medium thickness $\left(t_{e f f}\right)$, peak position $\left(E_{L S P R}\right)$, amplitude $\left(\epsilon_{2, \text { LSPR }}\right)$, and width $(F W H M)$ of the oscillator functions attributed to the LSPRs are $\left\{t_{\text {eff }}=59 \AA, E_{L S P R}=2.15 \mathrm{eV}, \epsilon_{2, L S P R}=1.71\right.$, and FWHM $=0.90 \mathrm{eV}\}$ and $\left\{t_{\text {eff }}=190 \AA, E_{\text {LSPR }}=1.83 \mathrm{eV}, \epsilon_{2, L S P R}=5.64\right.$, and $F W H M=1.28 \mathrm{eV}\}$ for panels (a) and (b), respectively. $E_{L S P R}$ and $\epsilon_{2, L S P R}$ are the coordinates indicated by the red circles. The scale bars equal $100 \mathrm{~nm}$.

rate of nucleation is limited by reaction kinetics rather than thermodynamics. Essentially, a higher conversion of $\mathrm{AuCl}_{4}{ }^{-}$to $\mathrm{Au}$ (III)-citrate complex in the solution is believed to aid the electron transfer required for $\mathrm{Au}$ nucleation and subsequent growth, and the $\mathrm{Au}(\mathrm{III})$ complex speciation is determined by the $c_{\text {citrate }}: c_{\mathrm{HAuCl}}$ ratio. The reason that the observed effect of this ratio is so pronounced in our experiments is likely that the chosen $c_{\text {citrate }}$ and $c_{\mathrm{HAuCl}}$ DoE levels are comparable in values. Obtaining rounder but smaller surface-supported NPs when using high $c_{\text {citrate }}: c_{\mathrm{HAuCl}}$ ratios is also consistent with having higher rates of nucleation and growth as adatom selectivity between different crystallographic planes and parasitic consumption of $\mathrm{Au}(\mathrm{III})$ is expected to decrease and increase, respectively. Citrate also has secondary effects, which might affect the final optical appearance of the growth media. First, negatively charged citrate ions adsorbed on the surface of NPs contribute to the electrostatic repulsion between NPs, reduce the rate of NP aggregation, and thus delay the redshift of the extinction peak. Second, it is conceivable that the adsorbed citrate could change the immediate dielectric environment of the NPs so as to influence optical extinction by the LSPR of NPs in solution. As for the effects of $c_{\mathrm{H}_{2} \mathrm{O} 2}$, our MLRs suggest that an increase does not affect the magnitude of the $\Delta I(t) / I_{0}$ change but expediates its onset and potentially also hasten its pace. The limited effects of $c_{\mathrm{H} 2 \mathrm{O} 2}$ is readily explained by the

fact that it is present in large excess throughout the DoE schemes.

We thus surmise that the rates of both nucleation and growth are kinetically limited. The latter is evident from the uneven deposition seen in our sample DoE scheme, which strongly suggests that mass transport of the $\mathrm{Au}(\mathrm{III})$ complex is the limiting factor.

3.3. Identification of a Suitable Seeded Growth Process Window. Heatmaps of our MLR-modeled $\bar{x}_{\text {Ellips }}$, $\bar{x}_{\text {Max FD }}, t_{0}$, and $K$ are shown in Figure 5 with the aim to visualize the growth process window in which quasi-spherical surface-supported NPs can be obtained and further indicate what we can expect in terms of particle size and extent of HN. Here, we have somewhat arbitrarily defined an array of quasispherical NPs as one having a $\bar{x}_{\text {Ellips }} \leq 1.2$. This leads to the process window partially shown in Figure 5 a, defined by $\left\{c_{\mathrm{H} 2 \mathrm{O} 2}\right.$ $\in[0.25,5.06] \mathrm{M}, c_{\mathrm{HAuCl}} \in[50,117] \mu \mathrm{M}$ and $c_{\text {citrate }} \geq 4.75$. $\left.c_{\mathrm{HAuCl} 4}-214 \mu \mathrm{M}\right\}$. The middle $c_{\mathrm{H} 2 \mathrm{O} 2}$ DoE level was used in plotting $t_{0}$ here, but equivalent plots of $t_{0}$ and $\tau$ for all three DoE levels are shown in Figure S20. The result shown in Figure 5a shows that an increase of $c_{\text {citrate }}$ (for a given $c_{\mathrm{HAuCl} 4}$ ) will result in more circular top-view cross sections, but from Figure $5 b$, it is observed that it will also lead to smaller NPs; the latter is due to increased $\mathrm{HN}$ in the growth medium with its affiliated parasitic consumption of $\mathrm{HAuCl}_{4}$. In Figure $5 \mathrm{c}$, the middle $c_{\mathrm{H} 2 \mathrm{O} 2}$ DoE level $(1.26 \mathrm{M})$ was used for plotting the $\mathrm{HN}$ onset time $t_{0}$ with consideration of gas bubble formation and reagent consumption. For completeness, citrate, in the absence of $\mathrm{H}_{2} \mathrm{O}_{2}$, does not seem to be able to reduce $\mathrm{Au}(\mathrm{III})$ to $\mathrm{Au}(0)$ and cause $\mathrm{HN}$ at an appreciable rate under these conditions; this was demonstrated by preparing an unseeded growth medium with a high $c_{\text {citrate }}: c_{\mathrm{HAuCl} 4}$ ratio but without any $\mathrm{H}_{2} \mathrm{O}_{2}$ and letting it run for $1 \mathrm{~h}$. As no change in $\Delta I(t) / I_{0}$ was observed, this suggests that $\mathrm{H}_{2} \mathrm{O}_{2}$ is the dominant reducing agent in this reaction system (Figure S21).

For verifying reproducibility, a cross-sample comparison of the four DoE CP replicates reveals virtually identical NPs, in terms of shape and size, although the array order has been maintained to a varying degree (Figure S22a-e,g). As for $\mathrm{HN}$ of the corresponding unseeded media, the curves fitted to $\Delta I(t) / I_{0}$ have similar rise times $(\tau)([45,60] \mathrm{s})$ and magnitudes $(K)([0.052,0.080])$ but more varied onsets $\left(t_{0}\right)$ $([196,381] \mathrm{s})$ as would be expected of a process containing stochastic elements (Figure S22f).

3.4. Seeded Growth without Agitation. One of the main takeaways from our DoE scheme is that consistent hydrodynamic conditions over the seed-decorated surface are required if a uniform degree of deposition is to be obtained in the probed reagent parameter space (Figure S2). The most straightforward way of approximating this is to forgo any growth medium agitation. As it is also of interest to determine to which extent $c_{\mathrm{H} 2 \mathrm{O} 2}$ can be dialed back, to minimize gas bubble formation and reagent consumption, we chose to run a sample series without agitation using the highest $\boldsymbol{c}_{\text {citrate }}(340$ $\mu \mathrm{M})$ and middle $c_{\mathrm{HAuCl}}(100 \mu \mathrm{M})$ DoE levels while progressively decreasing $c_{\mathrm{H} 2 \mathrm{O} 2}\{5.06 \mathrm{M}, 1.26 \mathrm{M}, 0.25 \mathrm{M}$, $0.025 \mathrm{M}\}$. To gauge the deposition uniformity, SEM images were acquired at the sample center as well as $100 \mu \mathrm{m}$ from the edge closest to the beaker bottom during SMNPG. The NPs at the edges were consistently somewhat larger than those in the sample center, which is partly to be expected due to higher levels of medium turbulence here during sample handling (Figure 6a). 

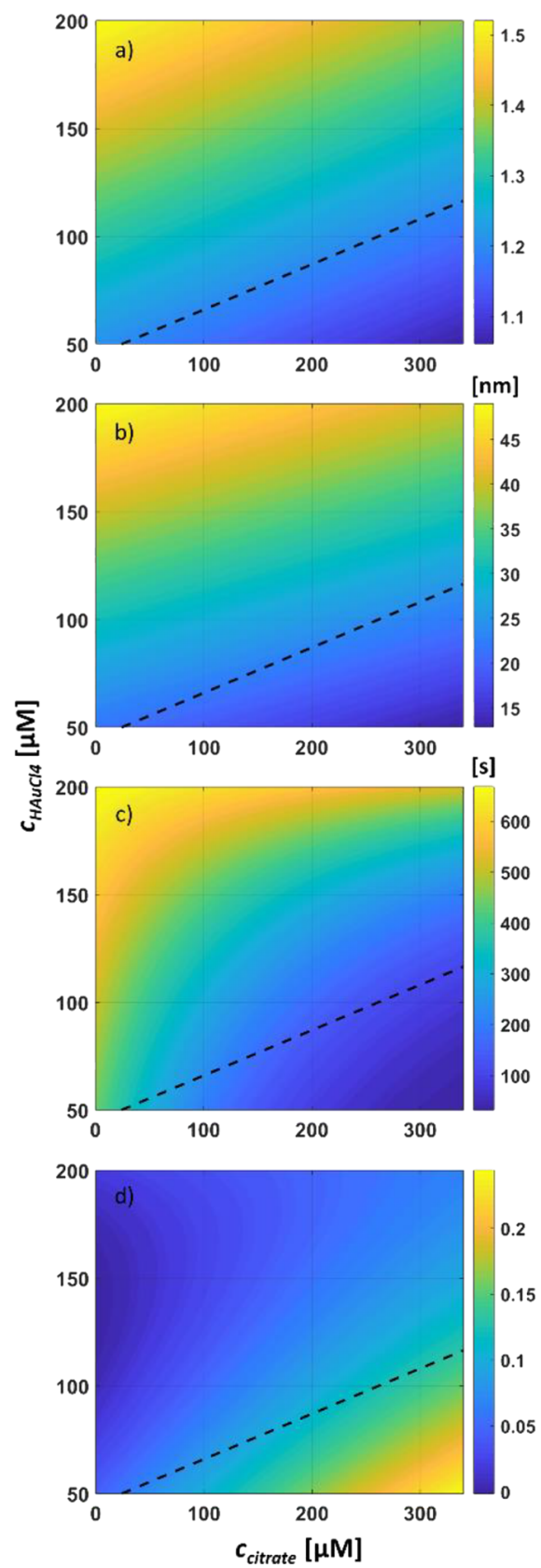

Figure 5. Heatmaps of modeled (a) NP mean ellipticity $\left(\bar{x}_{\text {Ellips }}\right)$, (b) $\mathrm{NP}$ mean maximum Ferret diameter $\left(\bar{x}_{\operatorname{Max} F \mathrm{D}}\right)$, unseeded medium $\Delta I(t) / I_{0}$ change, $(\mathrm{c})$ onset $\left(t_{0}\right)$, and $(\mathrm{d})$ magnitude $(K)$. The dashed lines represent $\bar{x}_{\text {Ellips }}=1.2$, thereby defining a process window for obtaining quasi-spherical NPs in the bottom right corner.

DIA suggests that the size and ellipticity of the NPs increase with decreasing $c_{\mathrm{H} 2 \mathrm{O} 2}$ (Figure 6b, Table S5), however, with the caveat that these samples differed slightly in size. The size difference was estimated by weighing the samples, assuming a uniform wafer thickness, and was found to be marginal $(<4 \%)$ (Table S5). Although using the highest $c_{\mathrm{H} 2 \mathrm{O} 2}$ yielded the most spherical NPs, one instance of gas bubble nucleation and growth was observed on the sample surface under these conditions, which could potentially be detrimental locally. All samples appear to be uniform after SMNPG under visual inspection (Figure S23 a-d), and SE optical characterization and feature extraction reveal well-defined LSPR peaks (Figure S24). We thus conclude that no agitation is preferable (in lieu a)

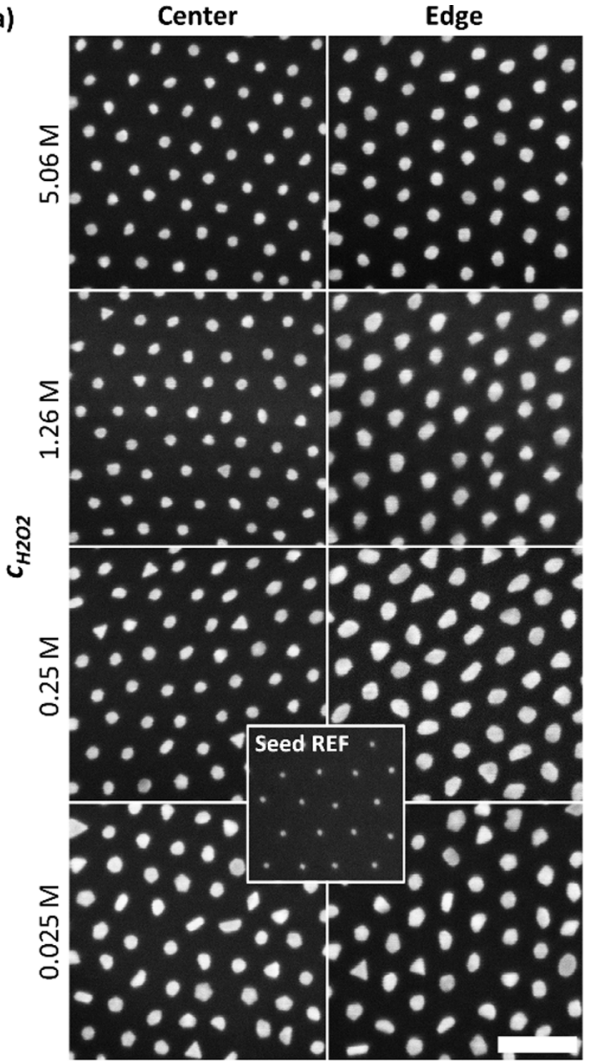

b)

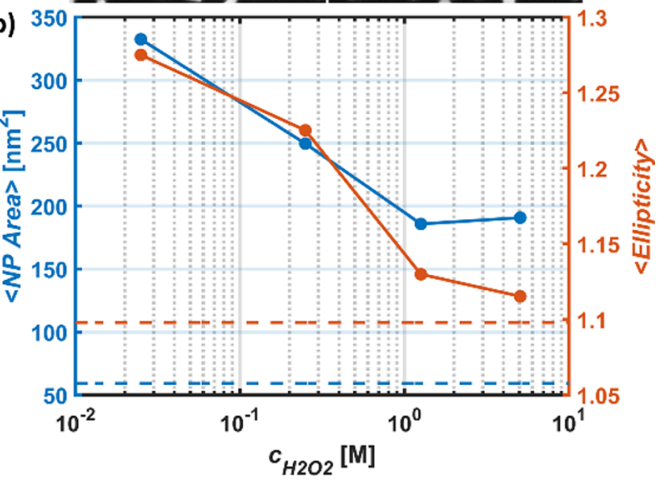

Figure 6. (a) Evaluation of progressively decreasing $c_{\mathrm{H} 2 \mathrm{O} 2}$ as shown in the figure in the absence of growth medium agitation. $c_{\mathrm{HAuCl}}=100$ $\mu \mathrm{M}$ and $c_{\text {citrate }}=340 \mu \mathrm{M}$ were used for all samples. The SEM images were acquired close to the sample center and $100 \mu \mathrm{m}$ from the sample edge, respectively. Scale bar equals $100 \mathrm{~nm}$. (b) Plots of the mean NP area and mean ellipticity of the surface-supported NPs (in sample center) on the samples in panel (a). Dashed lines represent the corresponding values for the initial seed arrays.

of more sophisticated means of ensuring uniform, well-defined hydrodynamic conditions) when using this SMNPG protocol. The result shown in Figure $6 \mathrm{~b}$ further suggests that $c_{\mathrm{H} 2 \mathrm{O} 2}$ might have a larger impact on the size and shape of the resultant NPs than our DoE scheme-based MLR models imply as $c_{\mathrm{H}_{2} \mathrm{O} 2}$ was not found to be a significant variable in either $\bar{x}_{\text {area }}$ or $\bar{x}_{\text {ellip }}$.

In addition to the experiments described above, we also investigated the effects of using PVA instead of, or in conjunction with, citrate in the growth medium. We found that PVA can delay the onset of the $\Delta I(t) / I_{0}$ change in the absence of seed particles and that larger surface-supported NPs can be obtained after seeded growth when using a combination of citrate and PVA. Addition of PVA appears capable of increasing the $\mathrm{Au}$ precursor utilization by suppressing $\mathrm{HN}$ in 
solution but at the cost of slightly reduced roundness (higher $\left.\bar{x}_{\text {Ellips }}\right)$ of the surface-supported NPs. The experiments are described in detail in the Supporting Information (Supporting Information Part II).

\section{CONCLUSIONS}

A seeded growth protocol consisting of a rapid, one-step procedure conducted at RT is employed on arrays of surfacesupported $\mathrm{Au}$ seed particles. With the goal to establish conditions for the growth of quasi-spherical NPs, a fully orthogonal DoE scheme is applied to identify a process window for concentrations of the three growth medium reagents, namely, $\mathrm{HAuCl}_{4}$, citrate, and $\mathrm{H}_{2} \mathrm{O}_{2}$ (i.e., $c_{\mathrm{HAuCl}}, c_{\text {citrates }}$ and $c_{\mathrm{H} 2 \mathrm{O} 2}$ ). Using SEM and DIA, various features related to the average size, shape, and array pattern quality are quantified for the resultant NP arrays. The extracted features are modeled as functions of the reagent concentrations using MLR with stepwise backward elimination of independent variables. By defining arrays of grown NP with a mean ellipticity $\leq 1.2$ as quasi-spherical, a suitable reagent concentration window is identified and defined by $\left\{c_{\mathrm{H} 2 \mathrm{O} 2} \in[0.25,5.06] \mathrm{M}, c_{\mathrm{HAuCl}} \in\right.$ $[50,117] \mu \mathrm{M}$ and $\left.c_{\text {citrate }} \geq 4.75 c_{\mathrm{HAuCl} 4}-214 \mu \mathrm{M}\right\}$. SE is further performed to study the optical properties of the resulting $\mathrm{Au} \mathrm{NP}$ arrays. Features related to the LSPR are identified and extracted, and their dependencies on reagent concentrations are also modeled using MLR.

In addition to reagent concentrations, we find that uniform hydrodynamic conditions over the NP-decorated substrate surface are key to obtain a macroscopically even deposition. This is presumably due to a mass-transport limited supply of the Au precursor in the surface reaction. Completely forgoing agitation of the growth medium is a convenient way of approximating the required conditions.

As varying degrees of parasitic $\mathrm{HN}$, seemingly dependent on the growth medium composition, were observed during the sample DoE scheme, the entire scheme was repeated using equivalent media without seeded substrates to map these tendencies. A proxy of the optical extinction in the media is tracked over time and then modeled in two steps so as to discern its dependencies on the growth medium composition. Increasing $c_{\text {citrate }}$, for a given $c_{\mathrm{HAuCl} \text {, }}$, is thereby found to exacerbate HN likely due to the ability of citrate to aid the electron transfer kinetics by acting as a complexing agent and thus affecting $\mathrm{Au}(\mathrm{III})$ speciation. This is unfortunate as a high $c_{\text {citrate }}$ also has a quasi-spherical shape-preserving effect on the surface-supported NPs. One potential solution to this issue is the addition of PVA to the growth medium as we observed that PVA can effectively delay the observable onset of our measured proxy for HN. Suppressed HN and thereby higher $\mathrm{HAuCl}_{4}$ utilization might thus be possible with PVA but appears to come at the cost of slightly more irregularly shaped surface-supported NPs.

We have identified seeded growth conditions that achieve a uniform deposition over large surfaces while maintaining the quasi-spherical shape and the initial array order of surfacesupported nanoparticles. Applications in catalysis, photonics, photovoltaics, plasmonics, and several other areas are conceivable.

\section{ASSOCIATED CONTENT}

\section{(s) Supporting Information}

The Supporting Information is available free of charge at https://pubs.acs.org/doi/10.1021/acs.langmuir.1c00693.
SEM images of the Au precursor-loaded BCP templates and seed particle arrays, tabulated feature values extracted via DIA and SE, compilations of photographs and SEM images of samples post-SMNPG, correlation matrix of dependent and independent variables, MLR models of extracted features, plots of the SE-modeled $\epsilon(E)$, plots of $t_{0}$ and $\tau$ for different $\mathrm{H}_{2} \mathrm{O}_{2}$ concentrations, unseeded DoE media side-by-side at specific timestamps, curve fits to $\Delta I(t) / I_{0}$ data from the DoE unseeded media, growth medium without $\mathrm{H}_{2} \mathrm{O}_{2}$, comparison of DoE CP replicates, SMNPG experiments using PVA instead of, or in conjunction with, citrate (PDF)

\section{AUTHOR INFORMATION}

\section{Corresponding Author}

Carl Hägglund - Division of Solar Cell Technology, Department of Materials Science and Engineering, Uppsala University, 75103 Uppsala, Sweden; (1) orcid.org/00000001-6589-3514; Email: carl.hagglund@angstrom.uu.se

\section{Authors}

Björn Landeke-Wilsmark - Division of Solar Cell Technology, Department of Materials Science and Engineering, Uppsala University, 75103 Uppsala, Sweden

Leif Nyholm - Department of Chemistry - Angström Laboratory, Uppsala University, 75120 Uppsala, Sweden

Complete contact information is available at: https://pubs.acs.org/10.1021/acs.langmuir.1c00693

\section{Notes}

The authors declare no competing financial interest.

\section{ACKNOWLEDGMENTS}

We like to express our appreciation to Lena Klintberg for fruitful discussion about statistical modeling. We further acknowledge the Swedish Research Council (Reg. No. 6212014-5599) and the Swedish Energy Agency (Project No. 45409-1) for financial support.

\section{ABBREVIATIONS}

$\begin{array}{ll}\text { ALD } & \text { atomic layer deposition } \\ \text { BCP } & \text { block copolymer } \\ \text { CP } & \text { centerpoint } \\ \text { DI } & \text { deionized } \\ \text { DIA } & \text { digital image analysis } \\ \text { DoE } & \text { design of experiment } \\ \text { HN } & \text { homogeneous nucleation } \\ \text { hPS } & \text { homopolystyrene } \\ \text { IP } & \text { inspection point } \\ \text { LSPR } & \text { localized surface plasmon resonance } \\ \text { NP } & \text { nanoparticle } \\ \text { RT } & \text { room temperature (21 }{ }^{\circ} \text { C) } \\ \text { sccm } & \text { standard cubic centimeters per minute } \\ \text { SEM } & \text { scanning electron microscopy } \\ \text { SMNPG } & \text { seeded metal nanoparticle growth } \\ \text { SVA } & \text { solvent vapor annealing } \\ \text { PS- } b \text {-P2VP } & \text { poly(styrene-block-2-vinylpyridine) } \\ \text { PVA } & \text { poly(vinyl alcohol) }\end{array}$

\section{REFERENCES}

(1) Navlani-Garcia, M.; Salinas-Torres, D.; Mori, K.; Kuwahara, Y.; Yamashita, H. Tailoring the Size and Shape of Colloidal Noble Metal 
Nanocrystals as a Valuable Tool in Catalysis. Catal. Surv. Asia 2019, 23, 127-148.

(2) Brus, L. Noble Metal Nanocrystals: Plasmon Electron Transfer Photochemistry and Single-Molecule Raman Spectroscopy. Acc. Chem. Res. 2008, 41, 1742-1749.

(3) Mejía-Salazar, J. R.; Oliveira, O. N. Plasmonic Biosensing. Chem. Rev. 2018, 118, 10617-10625.

(4) Langer, J.; Jimenez de Aberasturi, D.; Aizpurua, J.; AlvarezPuebla, R. A.; Auguié, B.; Baumberg, J. J.; Bazan, G. C.; Bell, S. E. J.; Boisen, A.; Brolo, A. G.; Choo, J.; Cialla-May, D.; Deckert, V.; Fabris, L.; Faulds, K.; García de Abajo, F. J.; Goodacre, R.; Graham, D.; Haes, A. J.; Haynes, C. L.; Huck, C.; Itoh, T.; Käll, M.; Kneipp, J.; Kotov, N. A.; Kuang, H.; Le Ru, E. C.; Lee, H. K.; Li, J.-F.; Ling, X. Y.; Maier, S. A.; Mayerhöfer, T.; Moskovits, M.; Murakoshi, K.; Nam, J.-M.; Nie, S.; Ozaki, Y.; Pastoriza-Santos, I.; Perez-Juste, J.; Popp, J.; Pucci, A.; Reich, S.; Ren, B.; Schatz, G. C.; Shegai, T.; Schlücker, S.; Tay, L.-L.; Thomas, K. G.; Tian, Z.-Q.; Van Duyne, R. P.; Vo-Dinh, T.; Wang, Y.; Willets, K. A.; Xu, C.; Xu, H.; Xu, Y.; Yamamoto, Y. S.; Zhao, B.; Liz-Marzán, L. M. Present and Future of Surface-Enhanced Raman Scattering. ACS Nano 2020, 14, 28-117.

(5) Atwater, H. A.; Polman, A. Plasmonics for improved photovoltaic devices. Nat. Mater. 2010, 9, 205-213.

(6) Jang, Y. H.; Jang, Y. J.; Kim, S.; Quan, L. N.; Chung, K.; Kim, D. H. Plasmonic Solar Cells: From Rational Design to Mechanism Overview. Chem. Rev. 2016, 116, 14982-15034.

(7) Hägglund, C.; Apell, S. P. Plasmonic near-field absorbers for ultrathin solar cells. J. Phys. Chem. Lett. 2012, 3, 1275-1285.

(8) Gutierrez-Rivera, L.; Peters, R. F.; Dew, S. K.; Stepanova, M. Application of EBL fabricated nanostructured substrates for surface enhanced Raman spectroscopy detection of protein A in aqueous solution. J. Vac. Sci. Technol., B 2013, 31, 06F901.

(9) Lee, S. W.; Lee, K. S.; Ahn, J.; Lee, J. J.; Kim, M. G.; Shin, Y. B. Highly Sensitive Biosensing Using Arrays of Plasmonic Au Nanodisks Realized by Nanoimprint Lithography. ACS Nano 2011, 5, 897-904.

(10) Vogel, N.; Fernandez-Lopez, C.; Perez-Juste, J.; Liz-Marzan, L. M.; Landfester, K.; Weiss, C. K. Ordered Arrays of Gold Nanostructures from Interfacially Assembled Au@PNIPAM Hybrid Nanoparticles. Langmuir 2012, 28, 8985-8993.

(11) Müller, M. B.; Kuttner, C.; König, T. A. F.; Tsukruk, V. V.; Förster, S.; Karg, M.; Fery, A. Plasmonic Library Based on SubstrateSupported Gradiential Plasmonic Arrays. ACS Nano 2014, 8, 94109421.

(12) Kim, H. J.; Lee, S. H.; Upadhye, A. A.; Ro, I.; Tejedor-Tejedor, M. I.; Anderson, M. A.; Kim, W. B.; Huber, G. W. Plasmon-Enhanced Photoelectrochemical Water Splitting with Size-Controllable Gold Nanodot Arrays. ACS Nano 2014, 8, 10756-10765.

(13) Cho, H.; Park, H.; Russell, T. P.; Park, S. Precise placements of metal nanoparticles from reversible block copolymer nanostructures. J. Mater. Chem. 2010, 20, 5047-5051.

(14) Mistark, P. A.; Park, S.; Yalcin, S. E.; Lee, D. H.; Yavuzcetin, O.; Tuominen, M. T.; Russell, T. P.; Achermann, M. Block-CopolymerBased Plasmonic Nanostructures. ACS Nano 2009, 3, 3987-3992.

(15) Cha, S. K.; Mun, J. H.; Chang, T.; Kim, S. Y.; Kim, J. Y.; Jin, H. M.; Lee, J. Y.; Shin, J.; Kim, K. H.; Kim, S. O. Au-Ag Core-Shell Nanoparticle Array by Block Copolymer Lithography for Synergistic Broadband Plasmonic Properties. ACS Nano 2015, 9, 5536-5543.

(16) Baruth, A.; Seo, M.; Lin, C. H.; Walster, K.; Shankar, A.; Hillmyer, M. A.; Leighton, C. Optimization of Long-Range Order in Solvent Vapor Annealed Poly(styrene)-block-poly(lactide) Thin Films for nanolithography. ACS Appl. Mater. Interfaces 2014, 6, 13770-13781.

(17) Glass, R.; Moller, M.; Spatz, J. P. Block copolymer micelle nanolithography. Nanotechnology 2003, 14, 1153-1160.

(18) Kastle, G.; Boyen, H. G.; Weigl, F.; Lengl, G.; Herzog, T.; Ziemann, P.; Riethmuller, S.; Mayer, O.; Hartmann, C.; Spatz, J. P.; Moller, M.; Ozawa, M.; Banhart, F.; Garnier, M. G.; Oelhafen, P. Micellar nanoreactors - Preparation and characterization of hexagonally ordered arrays of metallic nanodots. Adv. Funct. Mater. 2003, 13, 853-861.
(19) Lu, J. Q.; Yi, S. S. Uniformly sized gold nanoparticles derived from PS-b-P2VP block copolymer templates for the controllable synthesis of Si nanowires. Langmuir 2006, 22, 3951-3954.

(20) Landeke-Wilsmark, B.; Nyholm, L.; Hägglund, C. Seeded Growth of Large-Area Arrays of Substrate Supported Au Nanoparticles Using Citrate and Hydrogen Peroxide. Langmuir 2020, 36, 6848-6858.

(21) Lohmueller, T.; Bock, E.; Spatz, J. P. Synthesis of quasihexagonal ordered arrays of metallic nanoparticles with tuneable particle size. Adv. Mater. 2008, 20, 2297-2302.

(22) Diao, Z.; Kraus, M.; Brunner, R.; Dirks, J. H.; Spatz, J. P. Nanostructured Stealth Surfaces for Visible and Near-Infrared Light. Nano Lett. 2016, 16, 6610-6616.

(23) Kao, K. C.; Nishi, H.; Tatsuma, T. Effects of particle size and annealing on plasmon-induced charge separation at self-assembled gold nanoparticle arrays. Phys. Chem. Chem. Phys. 2018, 20, 37353740 .

(24) Hartling, T.; Seidenstucker, A.; Olk, P.; Plettl, A.; Ziemann, P.; Eng, L. M. Controlled photochemical particle growth in twodimensional ordered metal nanoparticle arrays. Nanotechnology 2010, 21, 145309.

(25) Ozdemir, B.; Seidenstucker, A.; Plettl, A.; Ziemann, P. Cyclic photochemical re-growth of gold nanoparticles: Overcoming the mask-erosion limit during reactive ion etching on the nanoscale. Beilstein J. Nanotechnol. 2013, 4, 886-894.

(26) Kruss, S.; Srot, V.; van Aken, P. A.; Spatz, J. P. Au-Ag Hybrid Nanoparticle Patterns of Tunable Size and Density on Glass and Polymeric Supports. Langmuir 2012, 28, 1562-1568.

(27) Sanchez-Iglesias, A.; Aldeanueva-Potel, P.; Ni, W. H.; PerezJuste, J.; Pastoriza-Santos, I.; Alvarez-Puebla, R. A.; Mbenkum, B. N.; Liz-Marzan, L. M. Chemical seeded growth of Ag nanoparticle arrays and their application as reproducible SERS substrates. Nano Today 2010, 5, 21-27.

(28) Lemineur, J.-F.; Ritcey, A. M. Controlled Growth of Gold Nanoparticles Preorganized in Langmuir-Blodgett Monolayers. Langmuir 2016, 32, 12056-12066.

(29) Lemineur, J.-F.; Schuermans, S.; Marae-Djouda, J.; Maurer, T.; Ritcey, A. M. Size-Modulation of Plasmonic Nanorings Obtained by the Self-Assembly of Gold Nanoparticles and Block Copolymers. J. Phys. Chem. C 2016, 120, 8883-8890.

(30) Liu, X. K.; Xu, H. L.; Xia, H. B.; Wang, D. Y. Rapid Seeded Growth of Monodisperse, Quasi-Spherical, Citrate-Stabilized Gold Nanoparticles via $\mathrm{H}_{2} \mathrm{O}_{2}$ Reduction. Langmuir 2012, 28, 1372013726.

(31) Li, Y. J.; Zhang, P. N.; Duan, J. L.; Ai, S. Y.; Li, H. S. One-step seeded growth of monodisperse, quasispherical, Tris-stabilized gold nanocrystals with sizes from 17 to $325 \mathrm{~nm}$. CrystEngComm 2017, 19, $318-324$.

(32) Sezgin, M.; Sankur, B. Survey over image thresholding techniques and quantitative performance evaluation. J. Electron. Imaging 2004, 13, 146-168.

(33) Young, D. S., Handbook of Regression Methods. 1st ed.; Chapman and Hall/CRC Press LLC: New York, 2017; pp. 1-654.

(34) Ross, M. B.; Mirkin, C. A.; Schatz, G. C. Optical Properties of One-, Two-, and Three-Dimensional Arrays of Plasmonic Nanostructures. J. Phys. Chem. C 2016, 120, 816-830. 